\title{
Hadronization via coalescence at RHIC and LHC
}

\author{
V. Minissale ${ }^{1,2}$, F. Scardina ${ }^{1,2}$ and V. Greco ${ }^{1,2}$ \\ ${ }^{1}$ Department of Physics and Astronomy, University of Catania, \\ Catania, Italy \\ ${ }^{2}$ INFN - Laboratori Nazionali del Sud, Catania, Italy
}

\begin{abstract}
An hadronization model that includes coalescence and fragmentation is used in this work to obtain predictions at both RHIC and LHC energy for light and strange hadrons transverse momentum spectra $(\pi$, $p, k, \Lambda)$ and baryon to meson ratios $(p / \pi, \Lambda / k)$ in a wide range of $p_{T}$. This is accomplished without changing coalescence parameters. The ratios $p / \pi$ and $\Lambda / K$ shows the right behaviour except for some lack of baryon yield in a limited $p_{T}$ range around $6 \mathrm{GeV}$. This would indicate that the AKK fragmentation functions is too flat at $p_{T}<8 \mathrm{GeV}$.
\end{abstract}

\section{Introduction}

In the study of the Quark Gluon Plasma partonic dynamics is not directly related to the observables measured in heavy ion collision experiments. The choice of the model for the hadronization process is a crucial point in order to have a comparison with experimental data. A decade ago the observation of the enhancement of baryon to meson ratio at intermediate transverse momenta and the scaling of elliptic flow according to the constituent quark number found in $\mathrm{Au}+\mathrm{Au}$ collisions at RHIC were a great surprise. The coalescence hadronization process can explain both this features that appears in the same intermediate $p_{T}$ region [1]. The coalescence approach born from the idea that in presence of QGP matter comoving partons combine their 
momenta to produce a final-state meson or baryon, this can happen at a time scale in which the partons rescatter and generate a thermal medium expanding with a collective radial flow [2]. Initial hard processes between colliding nucleons producing minijet partons provide a dominant contribution to hadron spectra at high transverse momentum.

\subsection{Coalescence model}

The coalescence approach is based on a Wigner function formalism. The hadron spectrum can be written as:

$$
\frac{d N_{H}}{d^{2} P_{T}}=g_{H} \int \prod_{i=1}^{n} \frac{d^{3} p_{i}}{(2 \pi)^{3} E_{i}} p_{i} \cdot d \sigma_{i} f_{q}\left(x_{i}, p_{i}\right) f_{H}\left(x_{i} . . x_{n}, p_{i} . . p_{n}\right) \delta^{(2)}\left(P_{T}-\sum_{i=1}^{n} p_{T, i}\right)
$$

where $f_{H}\left(x_{i} . . x_{n}, p_{i} . . p_{n}\right)$ is the hadron Wigner distribution function giving the probability for $n$ quark to form an hadron, $d \sigma$ denotes an element of a space-like hypersurface, $g_{H}$ is the probability of forming a color neutral object with the spin of the hadron considered from $n$ coloured quarks. The function $f_{q}(x, p)$ is the covariant distribution function of quarks (and antiquarks) in the phase space. For the light hadrons Wigner function we take a sphere in both space and momentum, with radii $\Delta_{r}$ and $\Delta_{p}$, respectively, which in the Wigner formalism are related by $\Delta_{r} \cdot \Delta_{p}=1$. For meson we have:

$$
f_{M}\left(x_{1}, x_{2} ; p_{1}, p_{2}\right)=\frac{9 \pi}{2\left(\Delta_{x} \Delta_{p}\right)^{3}} \Theta\left(\Delta_{x}^{2}-\left(x_{1}-x_{2}\right)^{2}\right) \Theta\left(\Delta_{p}^{2}-\left(p_{1}-p_{2}\right)^{2}+\left(m_{1}-m_{2}\right)^{2}\right)
$$

A good description of pion, kaon, proton, antiproton and $\Lambda$ spectra can be obtained with a radius parameter $\Delta_{p}=0.19 \mathrm{GeV}$ for mesons, $0.33 \mathrm{GeV}$ for proton, $0.38 \mathrm{GeV}$ for Lambdas [3].

\section{$2 \quad$ Fireball and model parameters}

In this study is considered a fireball of thermalized particles which includes light quarks, antiquarks and gluons at a temperature corresponding to $T_{c}=$ $160 \mathrm{MeV}$, that is about the cross-over transition temperature. Parton are distributed uniformly in the transverse plane. Longitudinal positions are distributed according to $z=\tau \sinh y$. Assuming a constant acceleration, we connect the radial expansion $R_{\perp}=R_{0}+0.5 \beta_{\max } \tau$ with the radial flow $\beta_{\max }$. In such manner the radial flow and the volume has constraints due to the 
experimental value of total multiplicity $d N / d y$ and total transverse energy $d E_{T} / d y$. The values obtained for this quantities are $\beta_{\max }=0.37, R_{\perp}=8.7$ $\mathrm{fm}, \tau=4.5 \mathrm{fm} / c$ at RHIC, and $\beta_{\max }=0.60, R_{\perp}=10.2 \mathrm{fm}, \tau=7.8 \mathrm{fm} / c$ at LHC. For their longitudinal momentum distribution we assume a uniform rapidity distribution in $y \in(-0.5,+0.5)$.

For partons in the quark-gluon plasma we take a thermal distribution for transverse momenta up to $p_{0}=2 \mathrm{GeV}$, in particular the momentum spectra for quark and antiquarks is given by:

$$
\frac{d N q, \bar{q}}{d^{2} r_{T} d^{2} p_{T}}=\frac{g_{q, \bar{q}} \tau m_{T}}{(2 \pi)^{3}} \exp \left(-\frac{\gamma_{T}\left(m_{T}-p_{T} \cdot v_{T} \mp \mu_{q}\right)}{T}\right)
$$

where $g_{q}=g_{\bar{q}}=6$ are the spin-color degeneracy of light quarks and antiquarks, and the minus and plus signs are for quarks and antiquarks, respectively. The slope parameter $\mathrm{T}$ is taken to be $T=165 \mathrm{MeV}$. Masses of light quarks and antiquarks are taken to be $m_{u, d, \bar{u}, \bar{d}}=300 \mathrm{MeV}$ and $m_{s, \bar{s}}=475 \mathrm{GeV}$, similar to the masses of constituent quarks. For the quark chemical potential we use a value of light antiquark to quark ratio which lead to the antiproton to proton ratio observed at midrapidity in heavy ion collisions experiments that is $\mu_{q}=10 \mathrm{MeV}$ at RHIC and vanishing at LHC. Partons at high transverse momenta (greater than $2 \mathrm{GeV}$ ) are mainly from the minijets produced in initial hard collisions among nucleons and are described by a power-law parametrization.

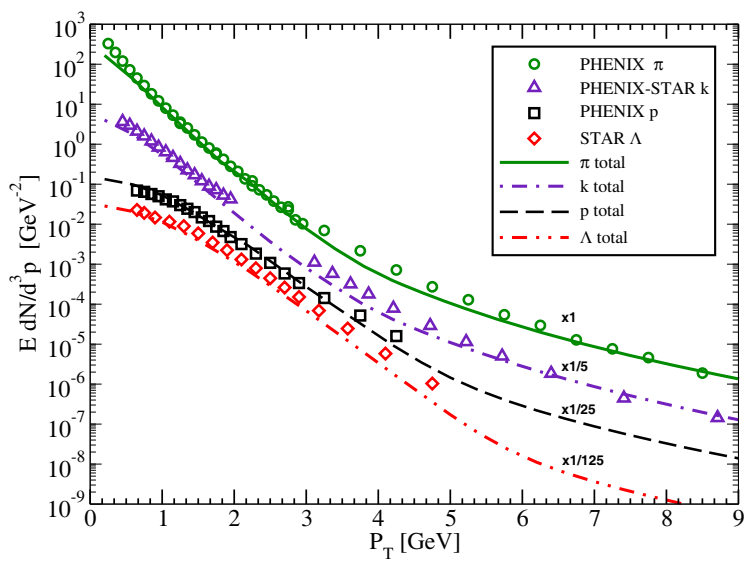

Figure 1: Particle transverse momentum spectrum at RHIC from $\mathrm{Au}+\mathrm{Au}$ collisions at $\sqrt{s}=200 \mathrm{GeV}$. Pion (solid), kaon (dash-dotted), proton (dashed), Lambda (dashed and double dotted). The spectra are scaled for better visibility. $[4,6]$ 


\section{Spectra}

In Fig. 1 are shown the transverse momentum spectra of pions, kaons, protons and Lambdas at RHIC. In all the spectra the contribution that comes from resonances decay are included with their decay channel and the pertinent branching ratios (e.g. for $\pi$ spectra a dominant contribution comes, in low momentum region, from $\rho$ decay). Higher resonances $\left(K^{*}, \Delta\right.$ in $\pi$ case) are less relevant and only contribute to some little better description at very low momenta [3]. We find a good description in a wide range of momentum. We can see in Fig.2 that the ratios $\bar{p} / \pi^{-}, \Lambda / K$ are well predicted, from the rise at low $p_{T}$ up to the peak region and then the falling-down behaviour. However in both cases it is clear that there is an underestimation in the predicted ratio in the region of $p_{T} \sim 5-7 \mathrm{GeV}$. Looking at the particle spectra this lack can be imputed to an insufficient production of baryons. In Fig. 3 are shown all the particle transverse momentum spectra at LHC. The results obtained in this case, occur without any change or addition of model parameters respect to the one at RHIC. Only the radial flow and volume of the hadronizing fireball are changed, and are constrained by comparison to the multiplicity and energy density experimentally measured.

There is a good agreement with the experimental data in all the transverse momentum region, except some lack of yield for pions at very low $p_{T}$ due to absence of all the possible resonances. The model is able to correctly predict the evolution, from RHIC to LHC, of the absolute yield and especially its $p_{T}$ shape. For baryons in the $p_{T}$ region where the fragmentation dominates,

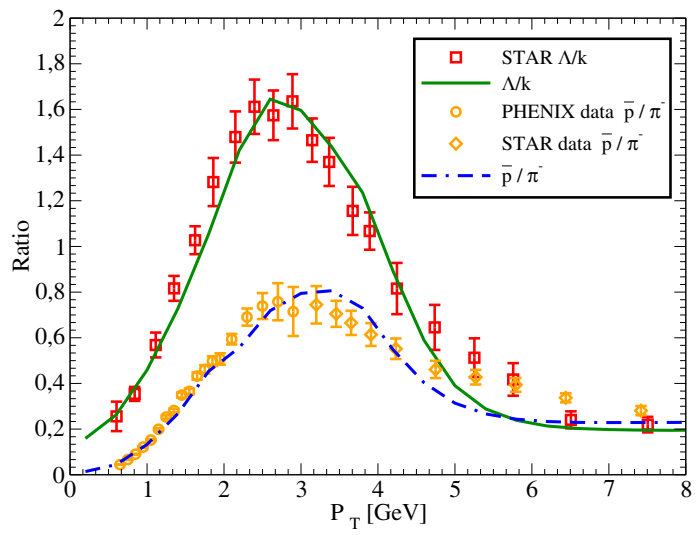

Figure 2: Antiproton to negative pion ratio (dash-dotted line) and Lambda to kaon ratio (solid line) at $\mathrm{RHIC}$ from $\mathrm{Au}+\mathrm{Au}$ collisions at $\sqrt{s}=200 \mathrm{GeV}$. [4,5]. 


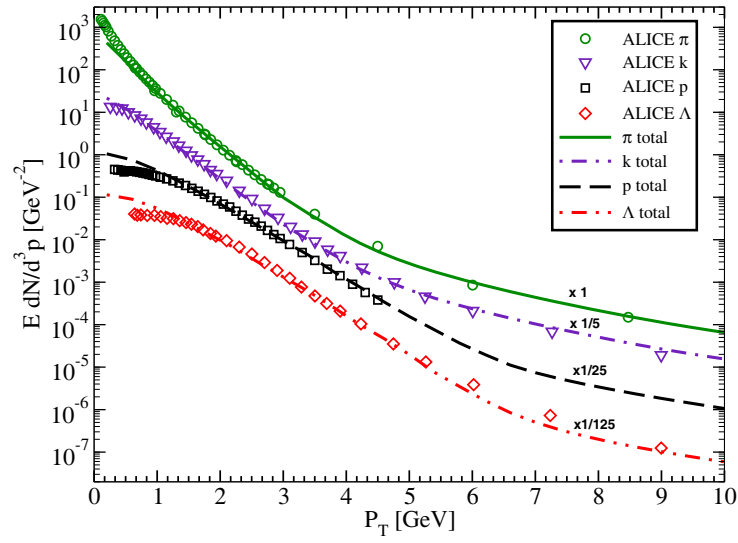

Figure 3: Particle transverse momentum spectrum at $\mathrm{LHC}$ in $\mathrm{Pb}+\mathrm{Pb}$ collisions at $\sqrt{s}=2.7 \mathrm{TeV}$. Pion (solid), kaon (dash-dotted), proton (dashed), Lambda (dashed and double dotted). The spectra are scaled for better visibility. $[6,7]$

i.e. $p_{T} \sim 6-7 \mathrm{GeV}$, there is some lack of yield. The baryon over meson ratios at LHC are shown in Fig.4. The model overestimate the ratio in the low $p_{T}$ region, due to the production excess of $p$ and $\Lambda$, but there is a good description especially in the peak region. These ratios show also that the region where fragmentation becomes dominant is characterized by an underestimation of baryons.

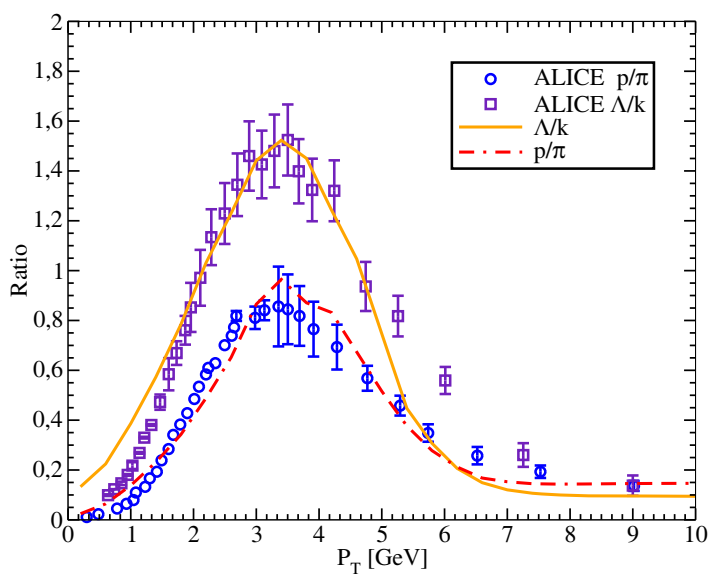

Figure 4: Proton to pion (dash-dotted line) and Lambda to kaon ratio (solid line) in $\mathrm{Pb}+\mathrm{Pb}$ collisions at $\sqrt{s}=2.7 \mathrm{TeV}$. [7] 


\section{Conclusions}

We have studied the evolution of the transverse momentum spectra from RHIC to LHC energy in a coalescence plus fragmentation approach. In order to predict the features at LHC no one adjustment of the coalescence parameter, like the width of Wigner function, has been made. The results obtained are in good agreement with the experimental data from LHC in a wide range of $p_{T}$. It appear that the independent fragmentation approach (at least using the AKK) gives too hard spectra at least up to $p_{T} \simeq 8 \mathrm{GeV}$ or it could be that coalescence contribution should extend to large $p_{T}$ with respect to the present modeling. There are studies that, recently, investigate a process that within the coalescence plus fragmentation approach could be quite important in solving this issue [8]. They essentially describe the inmedium fragmentation as a quark recombination of shower partons taking into account also the gluon splitting in quark pairs that recombine.

\section{References}

[1] STAR Collaboration, J. Adams et al., Phys.Rev. C72, 014904 (2005), nucl-ex/0409033.

[2] R. Fries, V. Greco, and P. Sorensen, Ann. Rev. Nucl. Part. Sci. 58, 177-205 (2008).

[3] V. Minissale, F. Scardina and V. Greco, arXiv:1502.06213, (2015), nucl-th.

[4] PHENIX Collaboration, S. Adler et al., Phys.Rev. C69, 034909 (2004), nucl-ex/0307022.

[5] STAR Collaboration, G. Agakishiev et al., Phys.Rev.Lett. 108, 072301 (2012), 1107.2955.

[6] ALICE Collaboration, B. Abelev et al., Phys.Rev.Lett. 109, 252301 (2012), 1208.1974.

[7] ALICE Collaboration,Abelev, Betty Bezverkhny and others, Phys.Rev.Lett. 111, 222301 (2013),1307.5530.

[8] K. C. Han, R. J. Fries, and C. M. Ko, J.Phys.Conf.Ser. 420, 012044 (2013), 1209.1141. 